EASD

Procedia
EURODYN 2020

XI International Conference on Structural Dynamics M. Papadrakakis, M. Fragiadakis, C. Papadimitriou (eds.)

\title{
USE OF THE DOMAIN REDUCTION METHOD TO SIMULATE THE SEISMIC RESPONSE OF AN EXISTING STRUCTURE PROTECTED BY RESONATING UNIT CELL METAMATERIALS
}

\author{
Constantinos Kanellopoulos ${ }^{1}$, Boris Jeremić ${ }^{2}$, Ioannis Anastasopoulos ${ }^{3}$ and Božidar \\ Stojadinović ${ }^{1}$ \\ ${ }^{1}$ ETH Zurich \\ Stefano-Franscini-Platz 5, 8093 Zurich, Switzerland \\ e-mail: \{kanellopoulos, stojadinovic\}@ibk.baug.ethz.ch \\ ${ }^{2}$ University of California, Davis \\ One Shields Avenue, Davis, CA 95616 \\ e-mail: jeremic@ucdavis.edu \\ ${ }^{3}$ ETH Zurich \\ Stefano-Franscini-Platz 5, 8093 Zurich, Switzerland \\ e-mail: ixa@igt.baug.ethz.ch
}

Keywords: Domain Reduction Method; Seismic Metamaterials; Resonating Unit Cells; Dynamic Soil-Structure Interaction

\begin{abstract}
Metamaterials have been successfully used during the past decade in the field of electromagnetism to control the propagation of electromagnetic waves. Interesting and possibly practically useful phenomena, such as negative refraction and generation of frequency bandgaps, may be induced in metamaterials. Recently, remarkable attempts have been made to bring this concept to the civil engineering scale in order to control the incoming seismic waves, mitigate their effects on structures and enhance seismic resilience of communities. A study on the potential of installing multiple resonating unit cells around an existing structure to improve its seismic performance is presented in this paper. Taking advantage of the $180^{\circ}$ phase difference that occurs right after the resonance of the unit cell masses, bandgaps can be generated at frequencies of interest for earthquake engineering applications. The Domain Reduction Method (DRM) is employed to realistically model the wave field (vertically propagating shear waves in this case). The model is verified and key features of DRM important for modeling the seismic response of metamaterials are exemplified.
\end{abstract}




\section{INTRODUCTION}

Use of metamaterials to control the propagation of electromagnetic waves was initially proposed by Pendry et al. [1]. Since then, many researchers applied this concept in different science and engineering fields, where the wavelengths are orders of magnitude larger, as in the case of mechanical waves in solids. In particular, earthquake engineers have considered locally resonant metamaterials or resonating unit cells or resonators (multiple unit cells with a vibrating mass inside each one of them), embedded into the soil as the means to guide the incoming seismic waves in certain frequency ranges (i.e., wave lengths) away from the structure to be protected. Most of these studies ([2], [3], [4], [5]) have shown, either numerically or experimentally, that this is feasible for the case of surface waves. Such waves couple with the resonators and are transmitted downwards before reaching the structure.

However, as shown by Zaccherini et al. [6], in case of more realistic inhomogeneous soil layers with depth-increasing stiffness, the initially downward-transmitted waves will eventually bend upwards and travel back towards the ground surface. This implies that the successfully diverted surface waves (aiming to protect one structure) may cause additional damage to neighboring structures. Such unintended "transfer" of seismic risk needs to be thoroughly addressed if seismic metamaterials are to find their way into civil engineering practice.

Most importantly, only a few studies ([7], [8]) have dealt with the other critical seismic wave component: the body shear waves. In contrast to surface waves, body waves propagate almost vertically through the soil, inciting the structure from below. A debate on which waves (surface or body) are more destructive is still ongoing in the earthquake engineering community. However, with a realistic model of the seismic wave field generated by the rupturing seismic fault, the relative importance of the different types of seismic waves can be assessed based on the basis of sound seismic risk analysis principles.

This study employs the Domain Reduction Method (DRM) ([9], [10]) that realistically models both body and surface waves. The DRM is a Finite Element (FE) analysis method that aims at reducing a large computational domain that includes the entire problem (including the seismic fault, the bedrock, the soil, and the structure), to a much smaller computational domain that only includes local soil and structure, using appropriate boundary and initial conditions, and interface layers [11]. The energy generated by oscillations of the structure is radiated back into the domain and dissipated using so-called damping elements at the DRM model boundary. DRM enables accurate modelling of all 6 components of seismic waves.

The paper focuses on a 2D (plane strain) domain, vertically propagating horizontal shear waves, and a structure represented by an elastic single degree of freedom (SDOF) system responding to horizontal base excitation, and metamaterial resonators similar with those ones proposed by Colombi et al. [7]. The aim is to showcase the effectiveness of the DRM in modelling soil-structure-metamaterial seismic response. Emphasis is placed on the importance of model verification and validation, and the illustration of some of the most powerful and useful features of the DRM, as implemented in the Real-ESSI Simulator [12].

\section{FINITE ELEMENT MODEL}

The in-plane linear elastic FE modelling is conducted using the Real-ESSI Simulator [12], in which the DRM is already incorporated and can be easily applied. The FE model is illustrated in Fig. 1, where the DRM elements are highlighted with orange color. The FE model is part of a one-to-two orders of magnitude larger domain that includes the rock and the remaining soil through which the seismic waves propagate. The seismic motions are inserted into the FE model by applying "effective" forces on the DRM elements. To calculate these effective forces, one needs to calculate the displacement and acceleration time histories for each node of the DRM 
elements using the free-field response of the larger domain and the known mass and elastic stiffness matrices of the FE model. This can be done either analytically ([13], [14]) or numerically, using one of the available commercial packages to calculate free-field response. This complex process is automated in Real-ESSI, obliviating the need to manually calculate the effective forces on DRM elements. In this way, the only necessary input is the acceleration and displacement time histories of the seismic motion.

A second important part of the FE model are the damping elements (shown with dark-grey color in Fig. 1), which surround the DRM elements (orange). Note the different damping ratio values assigned to the damper element zones on the sides and under the soil domain. The purpose of the damping elements is to damp out any waves generated by oscillations of the structure by artificially applying high values of Rayleigh viscous damping on such inciting waves. In the absence of a structure (free-field), the damping elements remain inactive, as there are no radiated waves entering their domain.

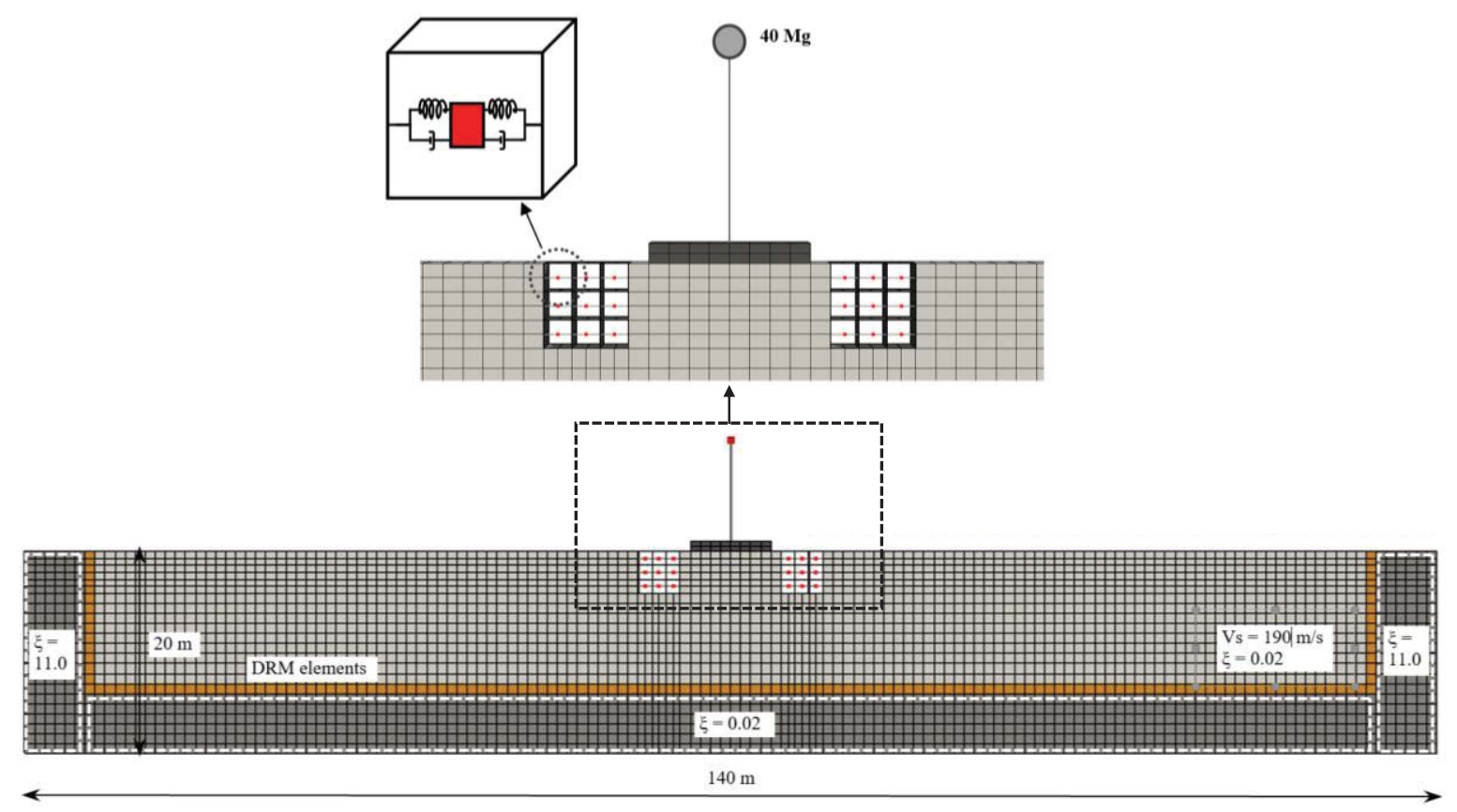

Fig. 1. Geometry of the FE model including the resonating unit cells and the structure.

\subsection{Soil, structure, and metamaterial properties}

A uniform and relatively soft soil layer of $20 \mathrm{~m}$ depth is considered, with $V_{s}=190 \mathrm{~m} / \mathrm{s}$ (shear wave velocity), $v=0.3$ (Poisson's ratio), $\rho=1.6 \mathrm{Mg} / \mathrm{m}^{3}$ (mass density), resting on rigid bedrock; the resulting horizontal natural period is $0.42 \mathrm{~s}$. The soil is modelled using 8-node elastic solid brick elements (2D modelling with 3D elements, i.e. a 3D slice), sufficiently discretized to properly capture wave field response (at least 10-elements per wavelength [15], [16]). Since rigid bedrock is assumed at $20 \mathrm{~m}$ depth, the radiated waves from the structure and the resonators should not be damped out by the damping elements at the bottom of the model. In this particular case, radiated waves propagating to the bottom boundary of the FE model should get fully reflected back towards the ground surface, and continue propagating (downwards and upwards), until they gradually dissipate or get artificially damped out by the lateral damping elements (this is why $\xi=0.02$ damping ratio is used for the damping elements in the horizontal zone under the soil, same as for the soil above, as shown in Fig. 1).

At this point, it should be noted that this is not how damping elements are supposed to be used. According to the DRM, damping elements should be used to damp out internally 
generated and radiated waves, not allowing them to go back into the main domain. A deeper FE model would be required for this purpose, also including the rock layer above the DRM elements, so that any radiated waves would be naturally reflected at the soil-rock interface.

An idealized SDOF system is used to represent an existing shear building, lying on a rigid surface foundation of $8 \mathrm{~m}$ width, which is modelled with 8 -node elastic brick elements. The SDOF system of $10 \mathrm{~m}$ height is modelled with elastic beam elements, assumed to be massless, and a $40 \mathrm{Mg}$ lumped mass on top. The stiffness of the beam is defined, so that the resulting natural period of the SDOF system is $0.45 \mathrm{~s}$. A Rayleigh viscous damping of 5\% was assumed.

The resonating unit cells are placed at the sides of the structure, embedded into the soil. Each unit cell (18 in total), is a hollow reinforced concrete (RC) box, containing a mass element (at the centre), connected to the sides of the box through two pairs of springs and dashpots, as shown in Fig.1. The RC box is modelled with elastic shell elements of $h=0.2 \mathrm{~m}$ thickness, Young's modulus $E=25 \mathrm{GPa}, \mathrm{v}=0.2$, and $\rho=2.5 \mathrm{Mg} / \mathrm{m}^{3}$, while a Kelvin-Voigt truss element is used to model each pair of springs and dashpots. The spring stiffness and the mass are determined so that the natural vibration period of each unit cell is $0.5 \mathrm{~s}-$ just above the $0.45 \mathrm{~s}$ period of the SDOF system (the reason of this selection will be explained in Section 2). Hence, assuming a mass of $2.5 \mathrm{Mg}$ for each unit cell, the spring stiffness should be $197.4 \mathrm{kN} / \mathrm{m}$. As discussed later on, the increase of the mass of the resonating unit cells leads to increase of their efficiency. Finally, a damping ratio of $6 \%$ is assumed for the unit cells.

Concerning the boundary conditions of the FE model, all degrees of freedom of the outer (lateral and bottom) boundaries of the damping elements are fixed. In addition, since a onecomponent ground motion excitation is employed (i.e., vertically propagating horizontal shear waves), the soil element nodes are allowed to move only horizontally. The same applies to the lumped mass at the top of the SDOF system and the vibrating masses inside the unit cells (i.e., no rotations or vertical motions are allowed). Finally, the foundation-soil and resonators-soil interfaces are modelled using full contact bonded elements.

\subsection{Input motion}

As shown in Fig. 2, the desired base excitation of the SDOF system is a Ricker pulse of 0.42 $\mathrm{s}$ period and $0.1 \mathrm{~g}$ peak amplitude. The input motion, applied on the DRM elements, is calculated to create such a wave field that will result in the targeted motion at the ground surface. Generation of the free-field wave field (by first performing deconvolution of the Ricker pulse down to the bedrock) to create the "effective" forces for the DRM elements is done automatically in Real-ESSI, using the analytical solutions described in [13] and [14].
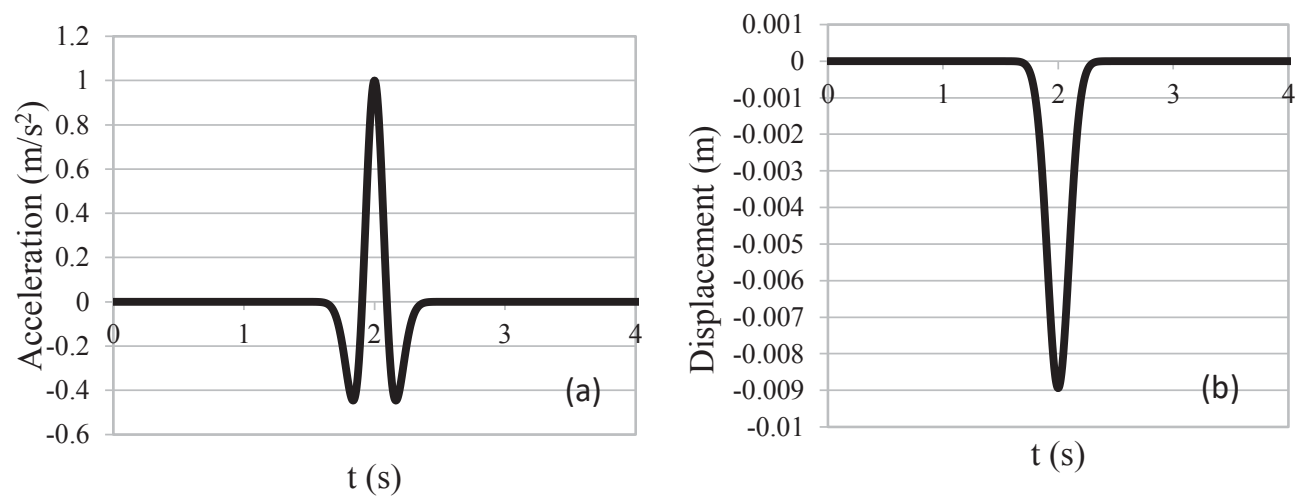

Fig. 2. Ricker pulse $\left(0.42 \mathrm{~s}, 1 \mathrm{~m} / \mathrm{s}^{2}\right)$ : (a) horizontal acceleration; and (b) horizontal displacement time histories. 


\subsection{Verification of the FE model}

\subsubsection{Free-field response}

The free-field model of Fig. 3 is developed and used to verify that the excitation at the ground surface is, indeed, the targeted Ricker pulse of Fig. 2. The developed free-field model is exactly the same as before, but the SDOF structure and the resonating cells are removed. Figure 4a depicts the deformed FE mesh with superimposed horizontal displacement contours for $t=2 \mathrm{~s}$. At this specific instant, the model surface attains the maximum negative displacement amplitude (-0.0089 m), as it should. Exactly the same deformed FE mesh is shown in Fig. 4b, but with exaggerated horizontal displacement scaling, to verify that the soil layer deforms in its first vibration mode shape, as it should, since the dominant period of the Ricker pulse $(0.42 \mathrm{~s})$ coincides with the natural period of the soil column $(0.42 \mathrm{~s})$.

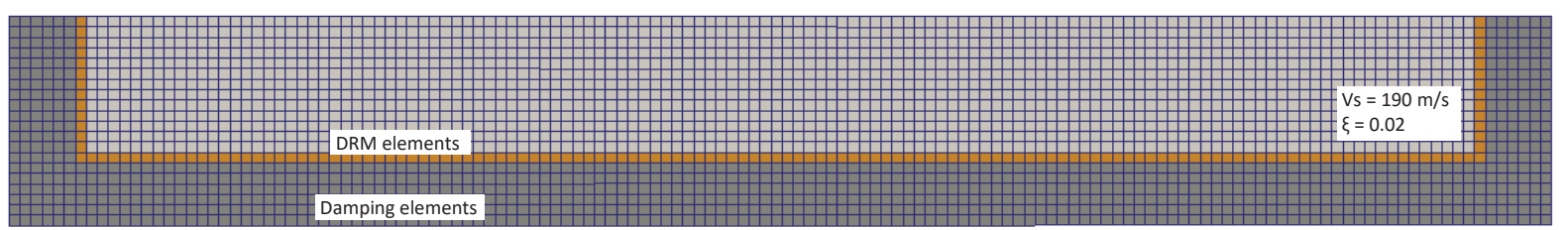

Fig. 3. FE model developed to verify the free-field response.

The effect of numerical damping is examined in more detail looking at the ground surface acceleration time histories (Fig. 5). Numerical damping can be tuned using the parameter $\gamma$ of the Newmark integration method [17]. At this point, it should be noted that numerical damping has nothing to do with the physical damping mechanisms. Such algorithmic damping was introduced by Newmark [17] in order to damp out the non-realistic high frequencies stemming from model discretization. Such numerical damping should be employed cautiously, as its improper use could also damp out the real response of the model.

(a)

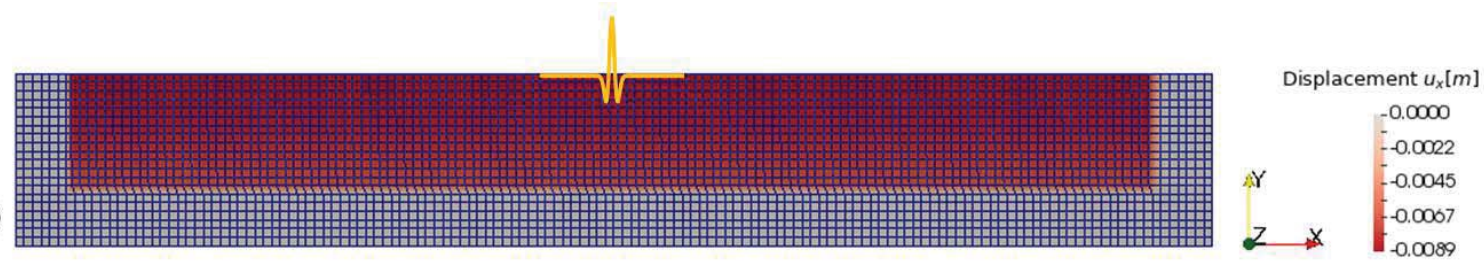

(b)

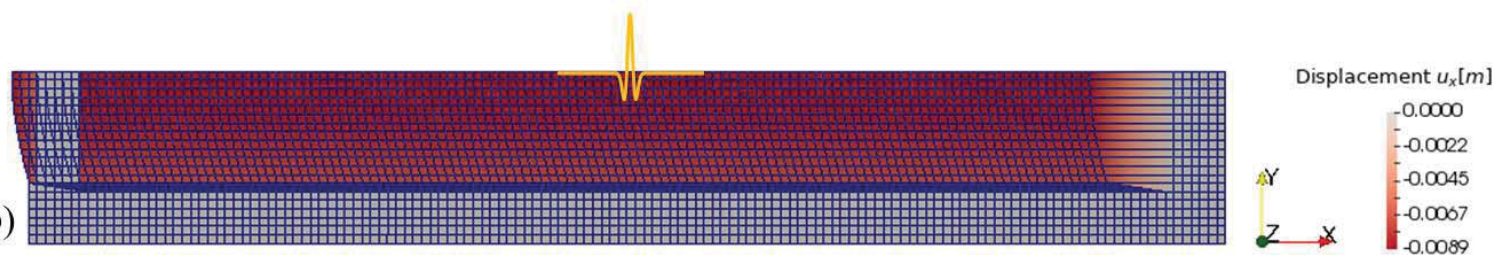

Fig. 4. Free field analysis - deformed FE mesh with superimposed horizontal displacement contours for $t=2 \mathrm{~s}$ :

(a) consistent scaling; (b) exaggerated horizontal displacements.

As shown in Fig. 5a, in the absence of numerical damping $(\gamma=0.5)$, the targeted Ricker wavelet is "contaminated" by spurious oscillations after the main pulse. These oscillations vanish with a slight increase of numerical damping $(\gamma=0.6)$, while the main pulse remains unaffected (Fig. 5b). Further increase to $\gamma=1.0$ leads to improper reproduction of the main pulse and to spurious oscillations (Fig. 5c). It is therefore essential to conduct a short sensitivity analysis at the beginning of a DRM study, to fine tune the necessary numerical damping. A 
similar sensitivity analysis is conducted for the complete model (including the SDOF structure and the resonators), concluding that no numerical damping is necessary (i.e., $\gamma=0.5$ ).
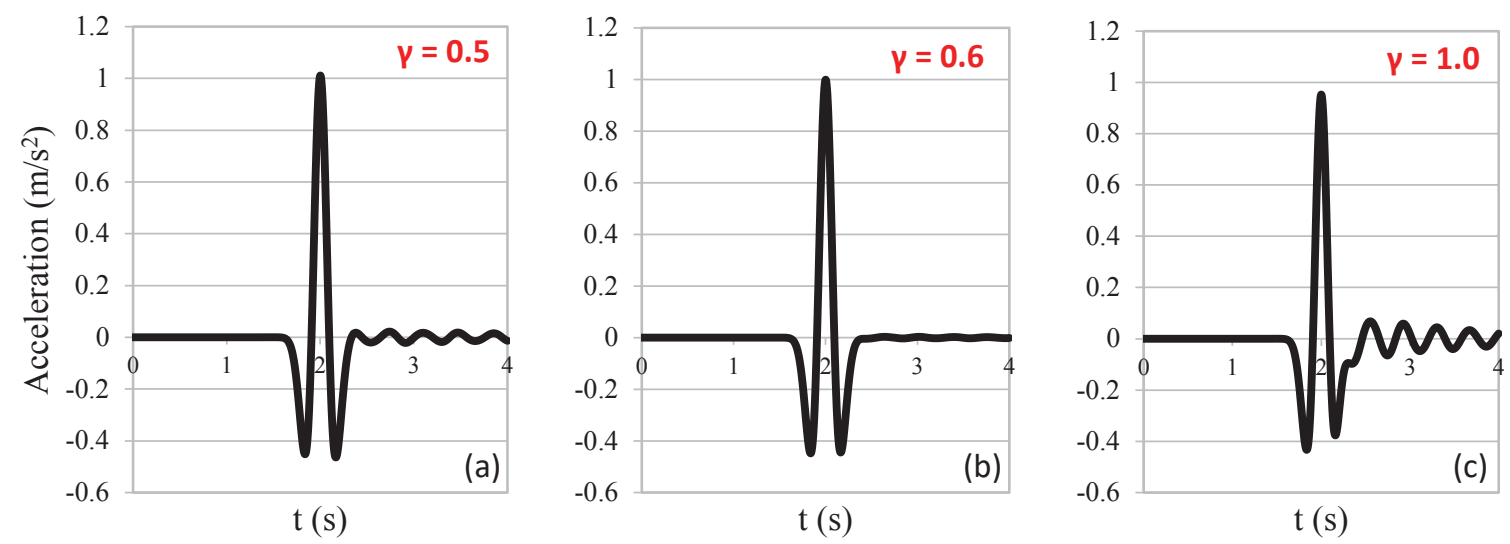

Fig. 5. Time histories of horizontal accelerations at the ground surface for three different values of numerical damping: (a) $\gamma=0.5$ (no numerical damping); (b) $\gamma=0.6$; and (c) $\gamma=1.0$

\subsubsection{Damping of the DRM elements}

As previously discussed, since the damping elements at the bottom of the FE model are not active, waves radiated from the structure can propagate downwards and upwards, being reflected at the bottom boundary (bedrock) and at the ground surface, respectively. The same Rayleigh viscous damping $(\xi=0.02)$ is used for the soil and the damping elements under the DRM elements, while a higher $\xi$ is employed for the lateral damping elements (Fig. 1).

A short sensitivity study is conducted to better understand the importance of damping elements. For this purpose, the Rayleigh viscous damping of all damping elements (including those under the DRM elements) is progressively increased until the response of the SDOF structure (in terms of horizontal accelerations at the mass) is not affected any more (Fig. 6). Such convergence is observed for $\xi=9(900 \%)$, as the response remains unaffected with further increase of damping to $\xi=11(1100 \%)$. This implies that, with $\xi=9$, all radiated waves from the SDOF structure and the unit cell resonators are fully absorbed by the damping elements.

Figure 7 compares the response for $\xi=11$ applied to all damping elements, to the case where the same high value of $\xi$ is applied only to the lateral damping elements. The acceleration time histories at the top of the SDOF structure are shown in Fig. 7a. Except from the non-negligible difference between the two cases, it can also be observed that that the structural response becomes more intense when the radiated waves are fully absorbed, which is not intuitive. As discussed later on, this is attributed to the response of the resonating unit cells. The vibrating masses inside the unit cells are moving out-of-phase with respect to the SDOF structure. As a result, the radiated waves from the resonators, propagate downwards to bedrock, being reflected back towards the ground surface. And since they are out-of-phase, this leads to a decrease of the acceleration of the SDOF structure. This is why the accelerations of the SDOF structure increase when the radiated waves are damped out. 


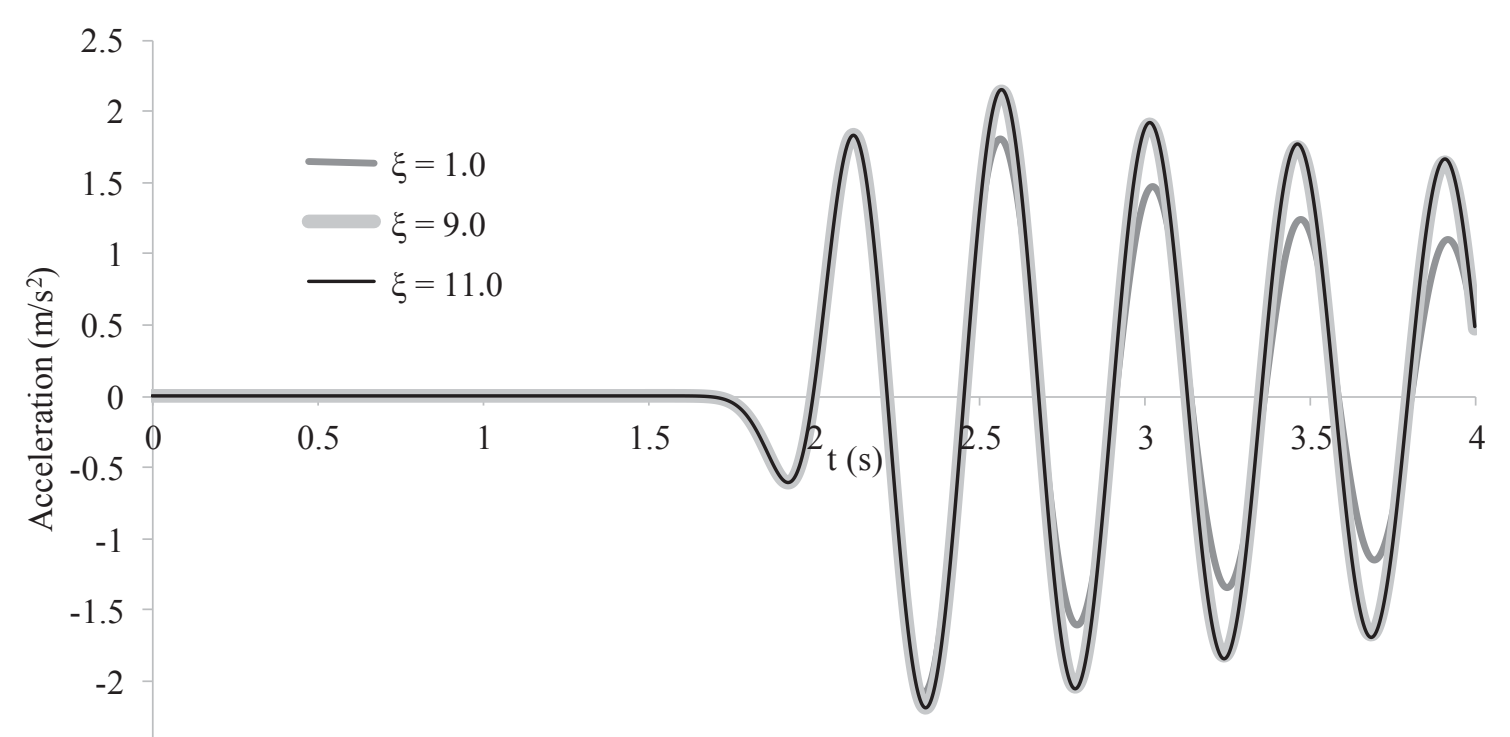

$-2.5$

Fig. 6. Time histories of horizontal acceleration at the top of the SDOF structure for three different values of damping ratio $(\xi=1,9$, and 11$)$ of the damping elements in the FE model.

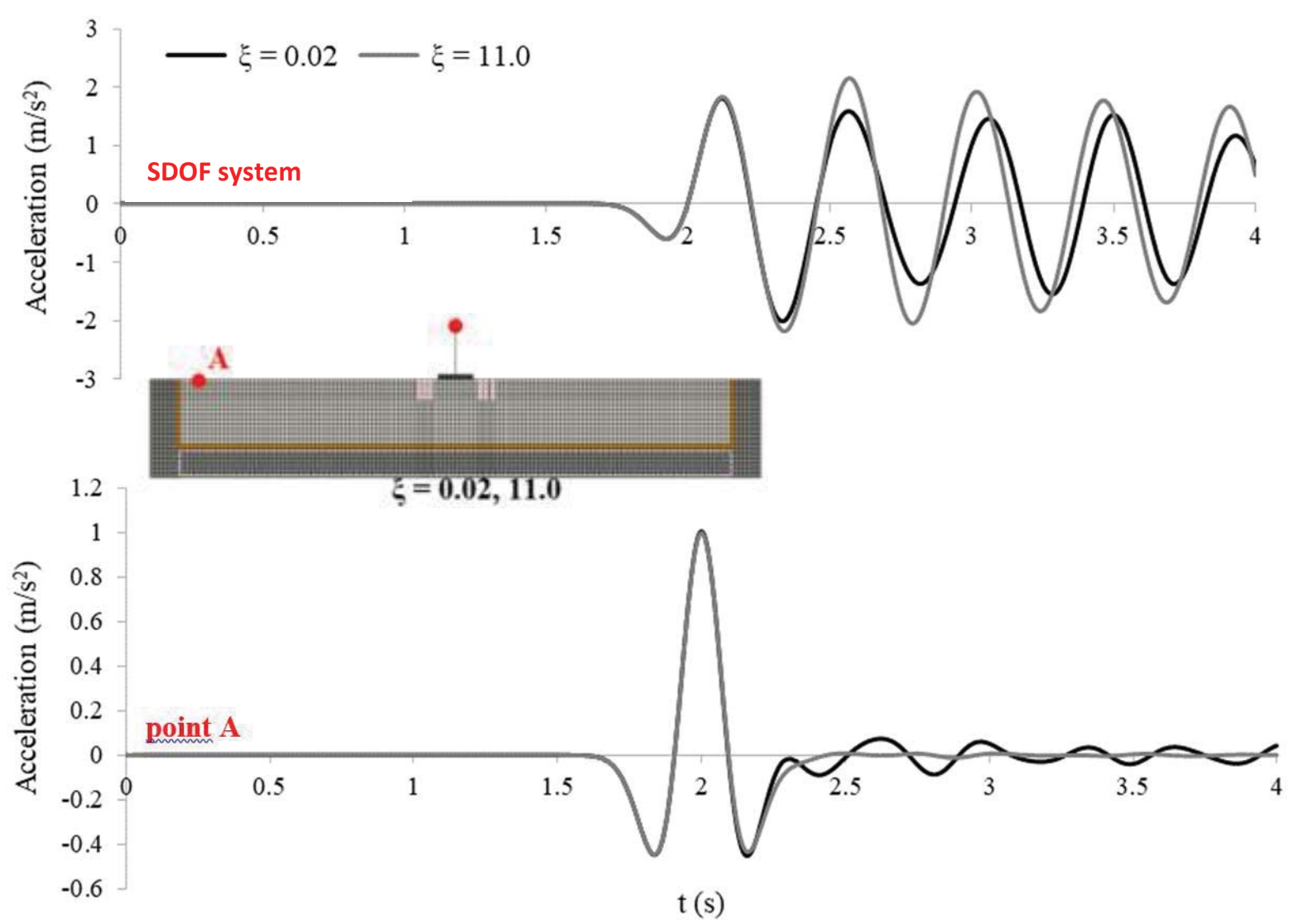

Fig. 7. Time histories of horizontal acceleration: (a) at the top of the SDOF structure; and (b) at the ground surface (point A). For the lateral damping elements, $\xi=11$ (i.e., laterally propagating waves are fully absorbed); for the damping elements under the DRM elements (inside the white box of the embedded figure), $\xi=11$ is compared to $\xi=0.02$ (i.e., practically no damping). 
Figure $7 \mathbf{b}$ offers a comparison of free-field ground surface accelerations (at Point A, away from the structure). When $\xi=11$ for all damping elements, the response at point $\mathrm{A}$ is the targeted Ricker pulse (free-field response). However, when only the lateral damping elements are "active", the radiated, from the structure and the resonators, waves are not damped, leading to the development of spurious oscillations after the main pulse. These waves will eventually be damped out when they reach the lateral damping elements. Ideally, those waves should have been able to continue propagating sideways, as shown in Fig. 8. The latter plots the FE model strain energy density for four different snapshots, showing the waves, generated by the vibration of the SDOF structure and the resonators, travelling laterally.

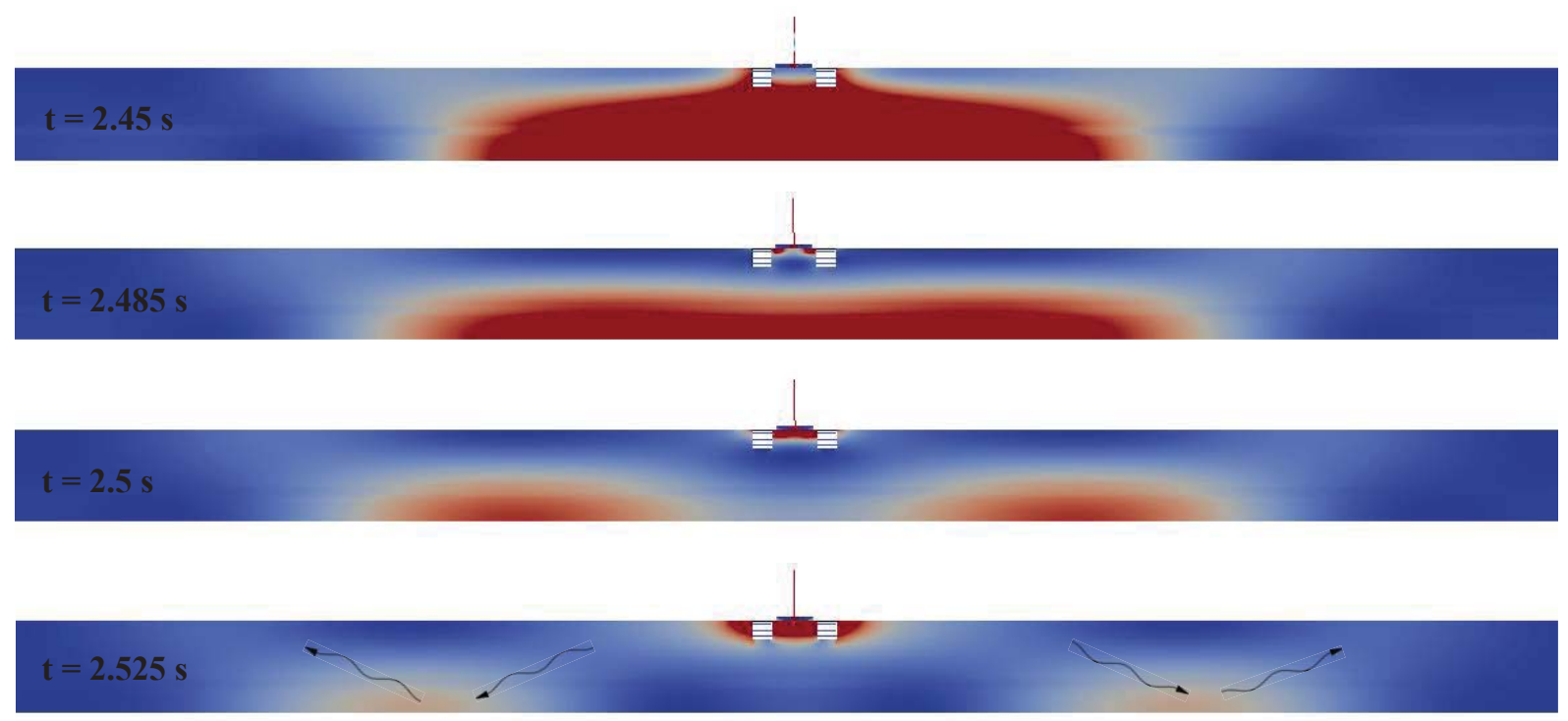

Fig. 8. Snapshots $(t=2.45,2.485,2.5$ and $2.525 \mathrm{~s})$ of strain energy density contours, showing the lateral propagation of waves radiated from the SDOF structure.

\subsubsection{Lateral boundaries of the FE model}

An additional sensitivity analysis is conducted to explore the effect of the FE model length. The length is gradually increased until the response of the SDOF structure is not affected any more. As shown in Fig. 9, this is achieved when the FE model length becomes equal to $780 \mathrm{~m}$, as further increase to $880 \mathrm{~m}$ does not seem to cause any changes in response. This leads to an extremely large FE model, which may be computationally affordable in 2D, but not in 3D. However, faster wave attenuation in 3D along with additional damping due to material nonlinearities [18] may allow significant reduction of the required length.

\section{RESULTS}

Before presenting some of the key results, it is important to explain a simple property of resonating unit cells that leads to decreased accelerations in the surrounding soil and, consequently, to a reduction of the seismic response of the structure. When a SDOF system resonates under harmonic base excitation, it moves with a $90^{\circ}$ phase difference with respect to the excitation displacement signal. If the harmonic excitation frequency is slightly changed, the displacement phase difference will rapidly drop to $0^{\circ}$ or jump to $180^{\circ}$, provided that the viscous damping ratio of the SDOF system is relatively low. In the FE model studied herein, the resonating unit cells have a slightly smaller resonant frequency $(2 \mathrm{~Hz}=1 / 0.5 \mathrm{~s})$ than the SDOF structure $(2.22 \mathrm{~Hz}=1 / 0.45 \mathrm{~s})$; the Ricker excitation is slightly larger $(2.38 \mathrm{~Hz}=1 / 0.42 \mathrm{~s})$. Thus, the resonant unit ce: 1 masses and the soil will move out-of-phase (with $180^{\circ}$ phase difference). 


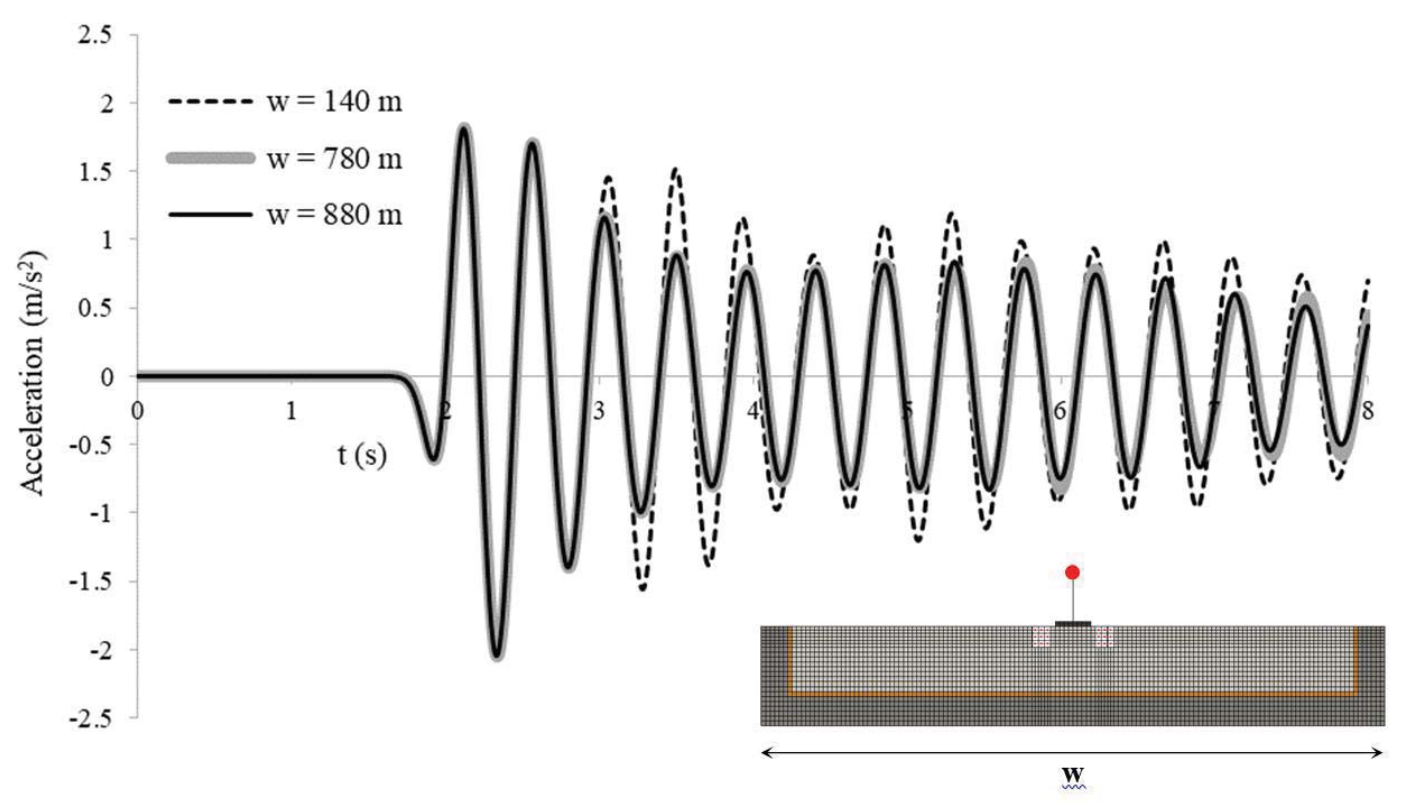

Fig. 9. Horizontal accelerations at the top of the SDOF structure for three different FE model lengths.

Such opposing motion will reduce the accelerations at the ground surface, leading to the desired reduction of the response of the SDOF structure. Out-of-phase resonator systems are more effective for continuous multi-cycle excitations, such as harmonics. The Ricker pulse was deliberately chosen (because of its similarity to the main pulse of recorded ground motions) to examine the efficiency of unit cell resonators for such short pulse signals.

Figure 10 compares the response of the SDOF structure, in terms of acceleration, with and without unit cell resonators. Two cases are examined with respect to the vibrating mass inside the unit cells: $2.5 \mathrm{Mg}$ and $5 \mathrm{Mg}$. The corresponding stiffness of the unit cell springs is appropriately adjusted, so that their natural period remains $0.5 \mathrm{~s}$. The peak response of the SDOF structure is barely affected by the unit cell resonators. A decrease in response can be observed only after the third cycle, revealing that the effectiveness of resonating unit cells increases with the number of excitation cycles.

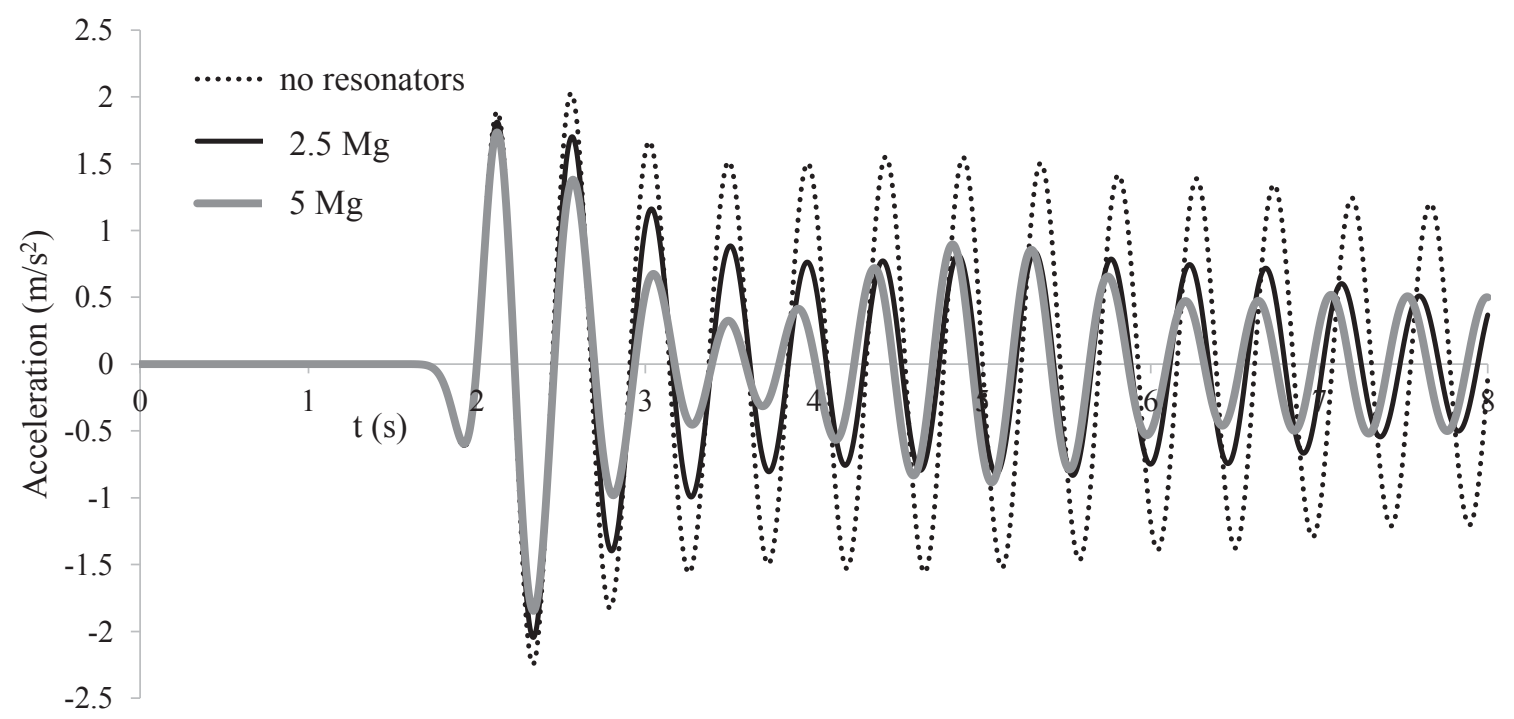

Fig. 10. Horizontal accelerations at the top of the SDOF structure with and without resonators. The vibrating mass in each unit cell is varied from to $2.5 \mathrm{Mg}$ to $5.0 \mathrm{Mg}$. 
Figure 11 compares the acceleration at the top of the SDOF structure to that of the unit cell vibrating mass $(2.5 \mathrm{Mg})$. Right after the end of the transient part (Ricker pulse), both the building and the resonators experience free vibration. Since the mass of the SDOF structure (40 $\mathrm{Mg})$ is approximately equal to the total mass of the resonators $(18 \times 2.5 \mathrm{Mg}=45 \mathrm{Mg})$, the inertia forces of the two system components (SDOF structure and resonators) will be proportional to their accelerations. The acceleration of the SDOF structure gradually decreases, reaching a minimum at $t=4 \mathrm{~s}$, when the resonators are moving exactly out of phase. For $t>5$ $\mathrm{s}$, the acceleration of the resonators is significantly reduced due to their internal viscous damping $(\xi=0.06)$. After this point, the free-vibrating SDOF structure becomes the excitation source for the resonators, which are henceforth excited at the natural vibration period of the SDOF structure $(0.45 \mathrm{~s})$. And since their natural vibration period of $0.5 \mathrm{~s}$ is a bit longer, their masses are, again, going to move out of phase with the SDOF structure. In this way, the resonators continue playing a protective role, reducing the response of the SDOF structure.

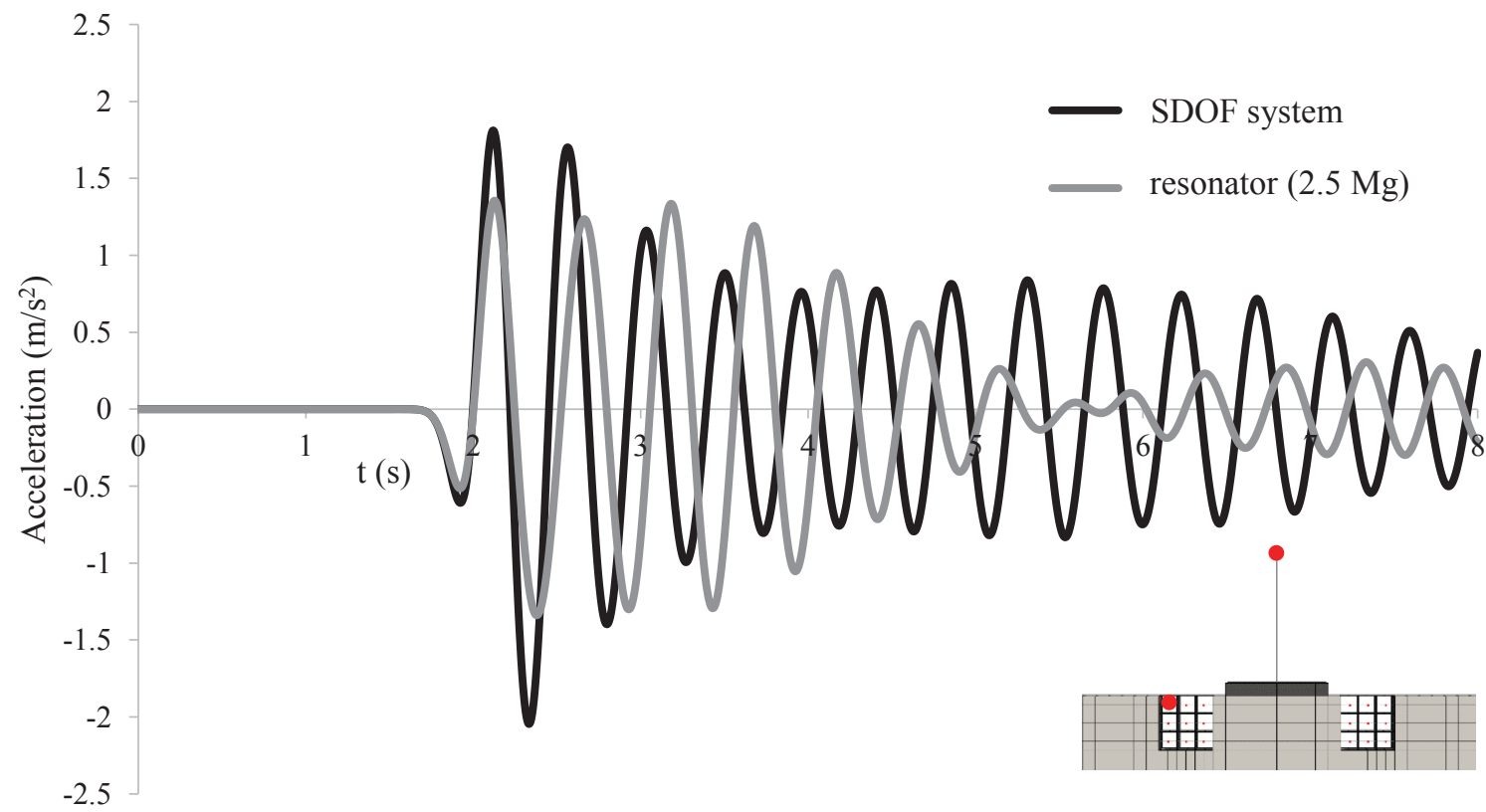

Fig. 11. Horizontal accelerations at the top of the SDOF system, compared to the mass of the top left resonator.

Figure 12 compares the horizontal displacement contours with and without resonators (of $2.5 \mathrm{Mg}$ unit cell mass) for a snapshot at $t=3 \mathrm{~s}$, when the systems are subjected to free vibration (same displacement scale factor). In the absence of resonators (Fig. 12a), the displacements are visibly larger compared to the case with $2.5 \mathrm{Mg}$ resonators (Fig. 12b), both for the soil and the SDOF structure. Observe also that the unit cell masses are moving in the opposite direction to that of the SDOF structure, which explains their protective function.

\section{CONCLUSIONS}

The paper has presented a preliminary study on the potential of installing multiple resonating unit cells around an existing structure to improve its seismic performance. The following key conclusions are summarized as follows:

- The Domain Reduction Method (DRM) was used within the Real-ESSI Simulator to realistically model both body and surface waves in FE models, and investigate soil-structuremetamaterial interaction. Some of its powerful features, such as the possibility to damp out waves radiating from the vibrating structures, were demonstrated. 
- The importance of conducting short sensitivity analyses to determine the parameters of the FE model was stressed. In this study, the sensitivity of the FE model to numerical damping and the length of the FE model were studied in detail. It was shown that (for this particular case) the lateral boundaries of the FE model should be placed at a considerable distance from the structure in order not to affect its response. It was also shown that adding numerical damping in the analysis to mitigate the spurious high-frequency response due to model discretization might also damp out real frequency components of response.

- The resonating unit cell metamaterials were effective in reducing the free-vibration response of an existing structure for the specific case analysed. It was shown that such metamaterials are effective thanks to the out-of-phase vibration of the unit cell masses with respect to the soil and the structure. This is achieved by tuning the resonator unit cell vibration periods to be slightly longer than the fundamental natural vibration period of the protected structure. Their effectiveness increases with increasing unit cell mass, provided they remain properly tuned.

(a)
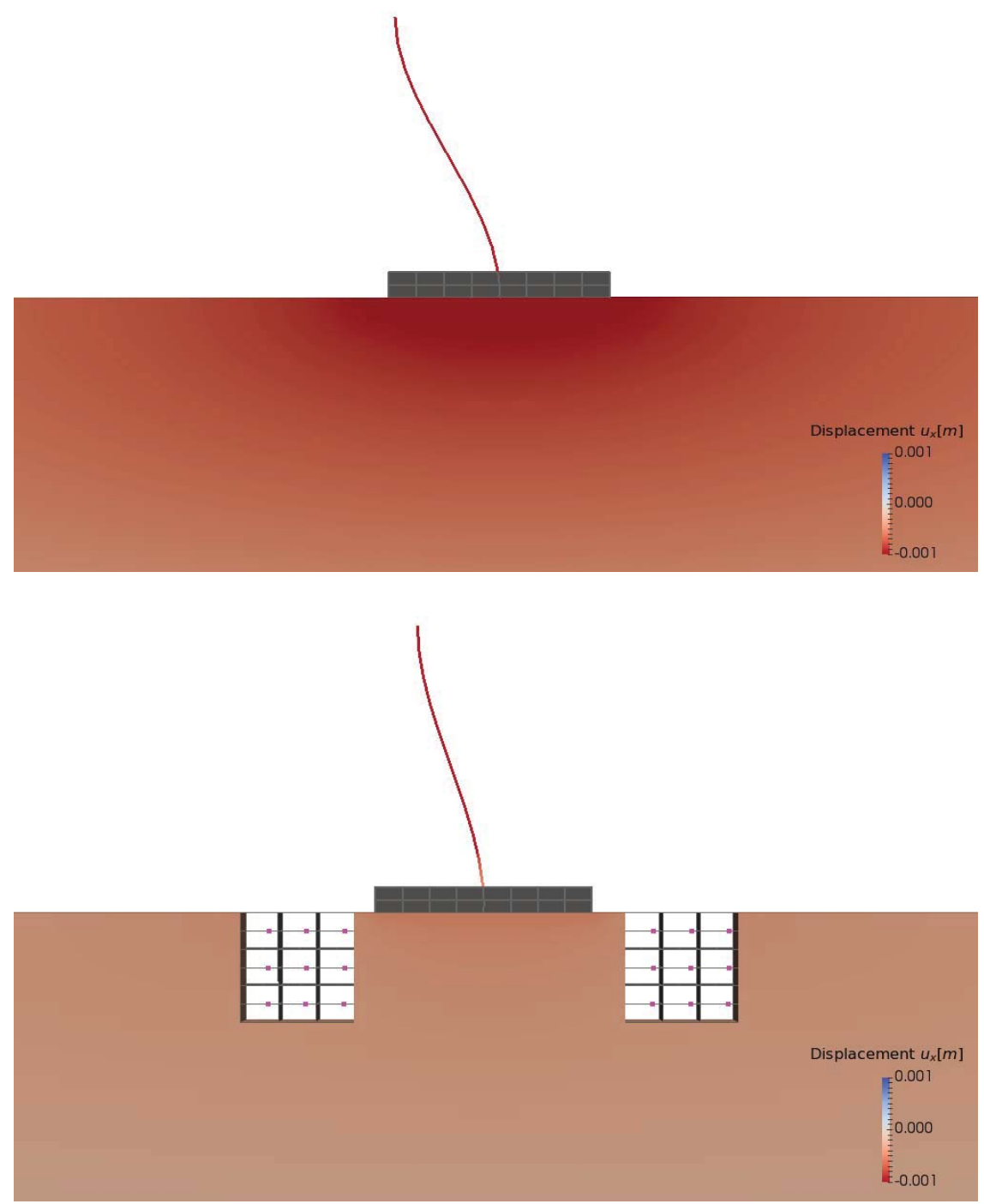

Fig. 12. Horizontal displacement contours: (a) no resonators; compared to (b) resonating unit cells (2.5 Mg). 


\section{Acknowledgements}

This work lies within the "INSPIRE" EU program. This project has received funding from the European Union's Horizon 2020 research and innovation program under grant agreement No 813424.

\section{REFERENCES}

[1] J. B. Pendry, D. Schurig and D.R. Smith. Controlling electromagnetic fields. Science 312, 1780-1782, 2006.

[2] A. Palermo, S. Krödel, A. Marzani and C. Daraio. Engineered metabarrier as shield from seismic surface waves. Scientific Reports 6, 1-10, https://doi.org/10.1038/srep39356, 2016.

[3] A. Palermo, S. Krödel, K. H. Matlack, R. Zaccherini, V. K. Dertimanis, E. N. Chatzi, A. Marzani, and C. Daraio. Hybridization of guided surface acoustic modes in unconsolidated granular media by a resonant metasurface. Phys. Rev. Appl. 9, 054026, 2018.

[4] A. Palermo, M. Vitali and A. Marzani. Metabarriers with multi-mass locally resonating units for broad band Rayleigh waves attenuation. Soil Dyn Earthq Eng 113:265, 2018.

[5] S. Krödel, N. Thomé and C. Daraio. Wide band-gap seismic metastructures. Extreme Mech Lett 4(September):111-117, 2015.

[6] R. Zaccherini, A. Colombi, A. Palermo , V. K. Dertimanis , A. Marzani, H. R. Thomsen, B. Stojadinovic and E. N. Chatzi. Locally resonant metasurfaces for shear waves in granular media. Phys. Rev. Appl. 13, 034055, 2020.

[7] A. Colombi, R. Zaccherini and A. Palermo. Mitigation of seismic waves: metabarriers and metafoundations bench tested. arXiv:1908.02056 [physics.geo-ph]

[8] P. Cacciola and A. Tombari. Vibrating barrier : a novel device for the passive control of structures under ground motion Subject Areas: Proc. R. Soc. A Math. Phys. Eng. Sci. 471, 2015 .

[9] J. Bielak, K. Loukakis, Y. Hisada and C. Yoshimura. Domain reduction method for threedimensional earthquake modelling in localized regions. part I: Theory. Bulletin of the Seismological Society of America, 93(2):817-824, $2003 \mathrm{a}$.

[10] C. Yoshimura, J. Bielak and Y. Hisada. Domain reduction method for three-dimensional earthquake modelling in localized regions. part II: Verification and examples. Bulletin of the Seismological Society of America, 93(2):825-840, 2003a.

[11] Jeremic et al. Nonlinear Finite Elements: Modelling and Simulation of Earthquakes, Soils, Structures and their Interaction. UCD and LBNL, CA, USA, (1989-2020) ISBN: 978-0-692-19875-9.

[12] Real-ESSI Simulator release version is Global Release, 20.01, Jan2020, http://realessi.us/

[13] W. T. Thomson. Transmission of elastic waves through a stratified solid medium. J. Appl. Phys., 21, 89-93, 1950. 
[14] N. A. Haskell. The dispersion of surface waves on multilayered media. Bulletin of the seismological Society of America, 43(1):17-34, 1953.

[15] J.-F. Semblat and A. Pecker. Waves and Vibrations in Soils: Earthquakes, Traffic, Shocks, Construction works. IUSS Press, first edition, 2009.

[16] K. Watanabe, F. Pisanò and Boris Jeremić. A numerical investigation on discretization effects on seismic wave propagation analyses. Engineering with Computers, 33(3):519545, Jul 2017.

[17] N. M. Newmark. A method of computation for structural dynamics. ASCE Journal of the Engineering Mechanics Division, 85, 67-94, July 1959.

[18] K. Kanellopoulos and G. Gazetas. Vertical static and dynamic pile-to-pile interaction in non-linear soil. Géotechnique, 70, No. 5, 432-447, https://doi.org/10.1680/jgeot.18.P.303, 2020 